Celik, A. 0.; Diplas, Panayiotis; Dancey, C. L. Instantaneous pressure measurements on a spherical grain under threshold flow conditions

Verfügbar unter / Available at:

https://hdl.handle.net/20.500.11970/99714

Vorgeschlagene Zitierweise / Suggested citation:

Celik, A. O.; Diplas, Panayiotis; Dancey, C. L. (2010): Instantaneous pressure measurements on a spherical grain under threshold flow conditions. In: Dittrich, Andreas; Koll, Katinka; Aberle, Jochen; Geisenhainer, Peter (Hg.): River Flow 2010. Karlsruhe: Bundesanstalt für Wasserbau. S. 755-762. 


\title{
Instantaneous pressure measurements on a spherical grain under threshold flow conditions
}

\author{
A. O. Celik \\ Department of Civil Engineering, Yunusemre Campus, Anadolu University, Eskisehir, 26470 Turkey \\ P. Diplas \\ Baker Environmental Hydraulics Laboratory, Department of Civil and Environmental Engineering, \\ Virginia Tech, Blacksburg, VA 24061, US \\ C. Dancey \\ Department of Mechanical Engineering, Virginia Tech, Blacksburg, VA 24061, US
}

\begin{abstract}
Despite the fact that the incipient motion of granular materials has been studied for over a century now, many questions remain unanswered about the actual mechanism responsible for entrainment of individual particles. Recent findings suggest that besides the instantaneous peak turbulent forces acting on individual grains, the durations over which these forces act have to be considered for determining the incipient motion of bed material. Force approximations using local flow velocity have received much attention and have been used extensively to investigate the role of turbulence on incipient motion. Direct measurements of instantaneous forces or pressures acting on grains, however, have not been undertaken as often because of the experimental difficulties involved. Yet, such direct measurements of hydrodynamic forces are essential. In this study we present results from flume experiments where instantaneous pressures acting on a fully exposed, spherical grain were measured simultaneously with local flow velocity in the vicinity of the particle. A spherical particle was instrumented with low-range, highly sensitive pressure transducers to measure the pressure simultaneously at its front, back, top and bottom. Drag and lift forces were estimated from the measured pressures acting on the test grain and the relationship between these forces (as well as individual pressures) with local flow velocity was examined. Experimental uncertainties in pressure measurements are also discussed.
\end{abstract}

\section{Keywords: Incipient motion, Hydrodynamic forces, Pressure measurements, Turbulence}

\section{INTRODUCTION}

In channel flow the local peaks of hydrodynamic forces acting on the rough sediment bed are responsible for particle movement near threshold flow conditions. Recent findings demonstrate that both the magnitude and the duration of these peak forces should be considered in incipient motion models (Diplas et al., 2008). Such models therefore require knowledge of the instantaneous local forces acting on the grains.

Einstein and El-Samni (1949) stated that two possible approaches could be used to obtain the hydrodynamic forces. One is to solve for the flow field over the bed surface and to employ statistical tools to calculate from this field the turbulence generated forces on the bed material. Although there are numerical models for solving a turbulent flow field over a randomly deposited and fully rough bed (for instance Stoesser et al. 2007), full resolution of the forces at realistic Reynolds num- bers is not yet entirely achievable. The second approach is to measure the forces or pressures directly acting on individual bed material.

Direct measurements of instantaneous forces or pressures acting on grains are uncommon however because of the experimental difficulties involved (e.g. refer to Hofland et al. 2005 and Detert et al. 2005).

Yet, such direct measurements of hydrodynamic forces are essential since the relationship between the instantaneous forces and other flow variables, such as streamwise velocity, is unclear. For example, Schmeeckle et al. (2007) conducted force measurements on natural and spherical particles and reported that the instantaneous drag force cannot be accurately predicted from the instantaneous local flow velocity using a constant drag coefficient, even though this method works well for the mean drag force. Instantaneous lift force, on the other hand is not correlated at all 
with the streamwise or vertical components of near-bed velocity according to the same study.

It is important to understand the effect of the bed configuration on the turbulent forces acting on the particles in the bed in order to build an accurate model. The turbulent flow field at a particular location and the resulting forces are affected by the upstream and local bed structure (Nelson et al. 1995, Hofland et al. 2005). More generally, regions of flow separation are often considered as one of the main contributors to the extreme pressures acting on the sediment grains.

At high Reynolds numbers the drag and lift forces come from the pressure distribution over the entire surface of the grain. As a result, the pressure fluctuations and their instantaneous distribution on the surface of a grain are directly relevant to the movement of bed material. In this regard, despite the fact that the force approximations are commonly used in force-balance based incipient motion models, there is a need for careful pressure measurements on rough beds for developing a more realistic incipient motion criterion.

In this study we present results from flume experiments where the instantaneous pressures acting on a fully exposed, spherical grain were measured simultaneously with local flow velocity in the vicinity of the particle. Drag and lift forces were estimated from the measured pressures.

\section{EXPRIMENTS}

\subsection{Devices and Methods for Experiments}

The experimental investigation was undertaken at the Baker Environmental Hydraulics Laboratory at Virginia Tech. The research flume employed is $20.5 \mathrm{~m}$ long, $0.6 \mathrm{~m}$ wide and $0.6 \mathrm{~m}$ deep. Pressure fluctuations near the bed were measured using a fixture attached to the flume bed. The fixture was located one meter upstream from the flume exit.

The fixture is an instrumented sphere, $12.7 \mathrm{~mm}$ in diameter secured on top of three identical size balls, arranged in a hexagonal densely packed structure. Solid brass spheres and brass tubes were fine-machined and used to construct the fixture. Pressure taps in the front (facing upstream), $p_{1}$; back, $p_{2}$; top, $p_{3}$; and bottom, $p_{4}$, of the instrumented sphere were connected to the pressure transducers fixed underneath the flume via a tubing system as shown in Fig. 1. The inner diameter of the brass tubing (and the pressure taps) is 1 $\mathrm{mm}$. We used Honeywell, Sensotec FP-2000 series, gage pressure transducers with a full pressure range of $25.4 \mathrm{~cm}$ of water column (corresponding to $5 \mathrm{~V}$ output), $0.1 \%$ accuracy and infinite resolution to measure the pressure fluctuations.

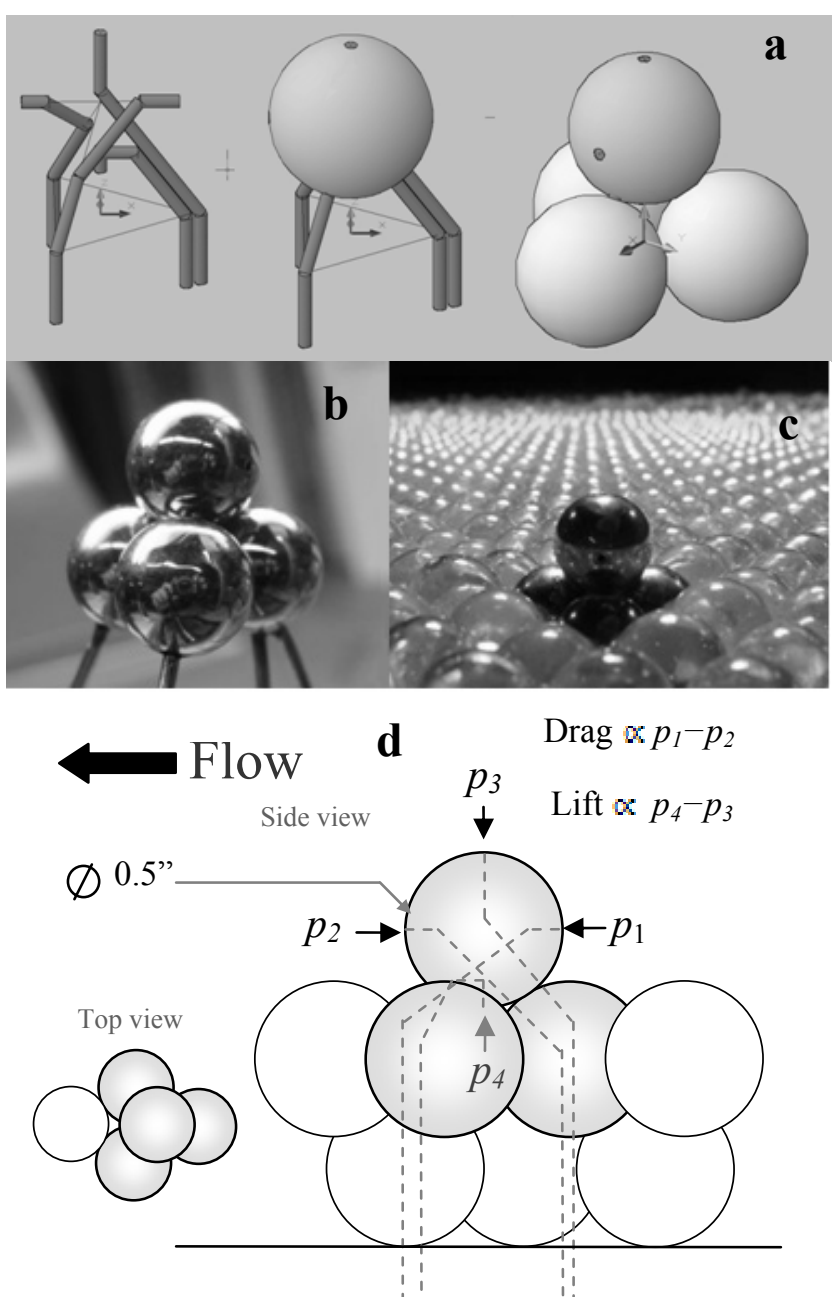

Figure 1. Instrumented fixture. a) Drawings of the fixture during design process. b) Photo of the fixture during assemblage. c) Photo of the fixture installed in the flume bed. d) Definition sketch for the pressure measurement points and bed geometry. Dashed lines indicate the tubing lines.

The fixture was designed, built and installed such that the tubing system was completely hidden within the instrumented grain and supporting base structure. The bed roughness (two layers of densely packed $12.7 \mathrm{~mm}$ balls) was also kept undisturbed and uniform in the test section as shown in Fig. 1c, extending over 2 meters upstream. Such simplified bed geometries, where a fully exposed spherical grain is resting on densely packed identical size spheres have been used by other researchers in sediment transport research (Dancey et al. 2002, Papanicolaou et al. 2002). But to the best of our knowledge, measurements of instantaneous pressures acting on a fully exposed spherical particle under turbulent flow conditions have never been carried out. Particularly unique here is the measurement of the surface pressure on the bottom of the sphere, simultaneously with the top, front and rear surface pressures. These types of measurements are essential to developing a better understanding of the instantaneous force components whose origin is the gradients of these pres- 
sures (Einstein and El-Samni, 1949, Scheemeckle et al. 2007).

Voltage outputs from the pressure transducers were recorded simultaneously with the local flow velocity measurements obtained with a 2-D laser Doppler velocimeter (LDV) system. The LDV measurement volume was located one diameter upstream of the instrumented grain along its centerline. The pressure and velocity signals were synchronized during the signal acquisition process via a TSI DL-100 external input module with a 16 bit $\mathrm{A} / \mathrm{D}$ converter. In this fashion, any unknown phase delay between the signals that may occur during the recording due to two separate data acquisition procedures was eliminated.

\subsection{Calibration of the Pressure Signal}

Pressure transducers were first calibrated under static loading. This was achieved by recording the voltage output from each transducer under known static pressures. Transducers were attached to the bottom of a Plexiglas box $(25.4 \mathrm{~cm} \times 25.4 \mathrm{~cm} \times 30$ $\mathrm{cm})$ and the static calibration was performed over the range of 0 to $23.6 \mathrm{~cm}$ of water column by measuring the output voltage of the transducers corresponding to various water levels in the box. The latter was measured carefully and used as the reference datum. This set-up is shown in Fig. 2.

The pressure transducers we used exhibited an initial condition (zero gauge pressure) offset voltage. These inherent DC offset voltages were also detected for each transducer before each static calibration, also before each flume test and taken into account. Consequently, a nearly perfect linear response was obtained yielding a static calibration factor of $196.85 \mathrm{mV} / \mathrm{cm}$ of water column for all transducers (coefficient of determination, $R^{2}=0.99$ ). Sampling rate and duration for individual static load tests were $250 \mathrm{~Hz}$ and $2 \mathrm{~min}$ respectively.

We employed various tubing arrangements in our pressure measurement set-up to connect the pressure taps and transducers as shown in Figs. 1a and $1 \mathrm{~d}$. Therefore, a dynamic calibration was necessary to evaluate the distortion, if any, in the pressure signal due to length of the tubes and bends. According to Yoshida et al. (2001) the effect of bends in a tubing line on the pressure signal is negligible if the effective sectional area of the tube at the bend zone is more than $50 \%$ of original area of the straight tube. The overall length of the tubing between the pressure taps and the transducer's sensing element in our experiments was no more than $10 \mathrm{~cm}$.

A dynamic calibration set-up similar to that reported by Yoshida et al. (2001) was used here. The Plexiglas box built for the static calibration tests was utilized again. This time we used a 10" subwoofer and an amplifier to generate a fluctuating pressure field in the sealed box. This setting was designed to produce very high-peak outputs. The subwoofer input consisted of white noise, generated by a MATLAB code. Refer to Yoshida et al. (2001) for more details on this dynamic calibration procedure.

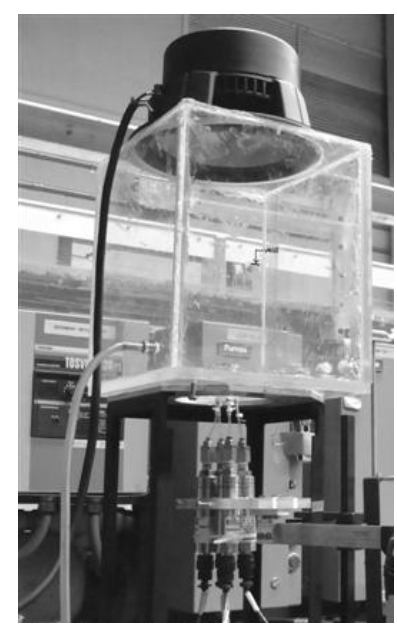

Figure 2. Test rig used for the static and dynamic calibration experiments.

The fixture was placed in the calibration box and the transducers were securely connected to the brass tubes underneath the box using flexible Teflon tubes (Fig. 2). This photo is useful as it represents the pressure transducer-brass tube connection in the actual flume tests as well. The transducers were held by a specially designed arm that was fixed to the lab floor without any connection to the calibration test platform. Flexible tubing was used to attach the transducers to the brass tubes (Fig. 2) to eliminate any vibration effects. The same arrangement was also used in the flume tests.

The frequency domain behavior of the tubing systems (e.g. for $p_{i}$ ) was investigated next. Pressures measured on top of the instrumented grain $\left(p_{3}\right)$ and those measured at the bottom of the grain $\left(p_{4}\right)$ were compared. These two measurement points were selected as $p_{3}$ exhibited the most compromising situation in terms of bending angles and $p_{4}$ had the sharpest bends in their tubing lines representing the near ideal and worst cases in the system respectively. That said and as it was not possible to precisely detect the input pressure generated in the box, the signal from $p_{3}$ was assumed as the input (undistorted signal) and $p_{4}$ was treated as output. In addition, the goal here with the dynamic calibration tests was not to detect the frequency response of the transducers but rather to identify the influence of tubing system on the pressure signals, with the assumption that the transducers we used were identical and the indi- 
vidual differences were negligible. The former was performed by the manufacturer and a flat frequency response up to $300 \mathrm{~Hz}$ was reported for the pressure transducers.

Dynamic calibration tests were performed using the test rig shown in Fig. 2, with water level of $3.8 \mathrm{~cm}$ in the box. We used a sampling rate of $1000 \mathrm{~Hz}$ and a sampling duration of $2 \mathrm{~min}$ in these tests. Subsequently, the transfer function between the two pressure signals measured at the top and bottom of the grain was estimated and the resulting amplitude and phase plots (Bode plot) between the two signals are given in Fig. 3.
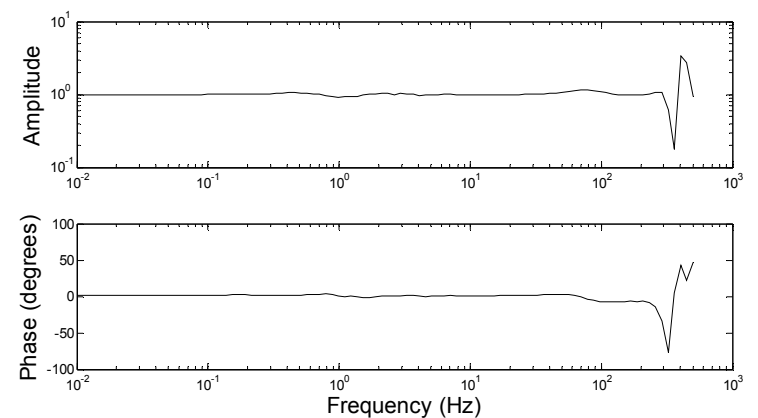

Figure 3. Amplitude and phase plots of transfer function between the pressure signals $p_{3}$ (top) and $p_{4}$ (bottom).

Figure 3 indicates that the tubing effect is negligible up to at least $200 \mathrm{~Hz}$, as the pressure signals measured using different tubing systems are regarded almost the same based on the obtained essentially flat frequency response.

Prior to the flume tests, a separate investigation was also carried out to detect the natural frequency of the flume (Stewart, 2009). Two Endevco Isotron Model 50 accelerometers were attached to the flume walls near the test section and vibration levels were recorded for a variety of conditions where the flume pump was on and also off. The results from this investigation indicate that our research flume has a natural frequency of around $100 \mathrm{~Hz}$ (when there is no pump and flow activity). The effect of the flume vibration due to the pump and the flow on the pressure signals was also found to be negligible as long as the transducers were fixed firmly but separate from the flume structure.

\section{RESULTS}

\subsection{Summary of the Flume Experiments}

Laboratory flume experiments were performed under nine uniform flow conditions and the bed configuration shown in Figs. 1c and d. Table 1 gives a summary of the flow conditions tested. These flow arrangements represent near threshold conditions for a Teflon ball with specific gravity of 2.3 identical in size to that of the instrumented grain (see Shields stresses in Table 1, column 5).

Table 1. Summary of the flow conditions

\begin{tabular}{|l|l|l|l|l|}
\hline Run & $\begin{array}{l}\text { Depth average } \\
\text { velocity, } U \\
(\mathrm{~m} / \mathrm{s})\end{array}$ & $\begin{array}{l}\text { Flow } \\
\text { depth, } h \\
(\mathrm{~cm})\end{array}$ & $R e^{*}$ & $\begin{array}{l}\text { Shields } \\
\text { Stress, } \tau^{*}\end{array}$ \\
\hline A1 & 0.47 & 8.1 & 438 & 0.011 \\
\hline A2 & 0.45 & 7.5 & 424 & 0.011 \\
\hline A3 & 0.43 & 8.2 & 413 & 0.010 \\
\hline A4 & 0.41 & 7.9 & 398 & 0.010 \\
\hline A5 & 0.42 & 8.3 & 385 & 0.009 \\
\hline A6 & 0.40 & 8.6 & 377 & 0.009 \\
\hline A7 & 0.41 & 9.1 & 372 & 0.008 \\
\hline A8 & 0.39 & 8.7 & 364 & 0.008 \\
\hline A9 & 0.35 & 8.9 & 330 & 0.007 \\
\hline
\end{tabular}

The sampling rate and the sampling duration for each run were $250 \mathrm{~Hz}$ and $15 \mathrm{~min}$ respectively. Individual pressure signals from all runs were checked for drift after each run which was found to be negligible in all cases. Based on the results from the investigations of measurement uncertainties presented earlier, all the pressure data from flume tests were first low-pass filtered with a cutoff frequency of $90 \mathrm{~Hz}$.

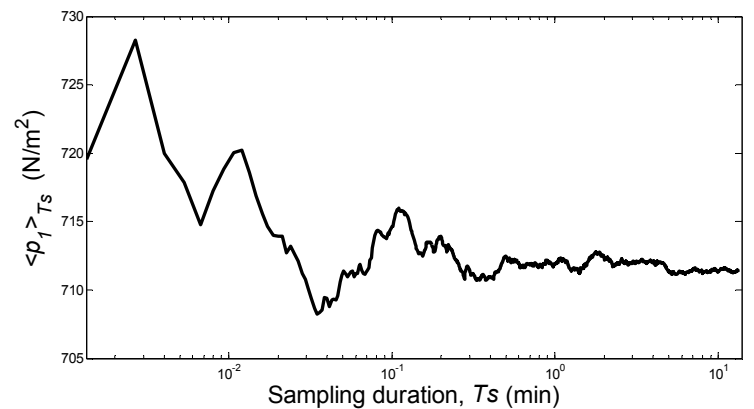

Figure 4. Time average pressure over the sampling duration $T s,<p_{T S}>$ vs. the sampling duration $T s$.

Influence of sampling duration was also examined in post-processing by subdividing the $15 \mathrm{~min}$ long pressure data into several shorter duration time series. Time average pressure values of each one of these time series were determined. Results of this analysis using $p_{1}$ data from run $\mathrm{A} 1$ are shown in Fig. 4. Noticeable variations in average pressure values are observed within the first $30 \mathrm{~s}$ of sampling period. In addition, a minimum of $1 \mathrm{~min}$ sampling duration is required to reduce the margin of sampling errors to a fraction of $1 \%$ in the mean pressure values. The same behavior was observed in data from all runs and this finding suggests that shorter sampling durations for pressure measurements will introduce considerable errors in statistics of the pressure data.

Figure 5a provides a comparison of measured time average pressures with static pressures at the same level obtained from measured flow depths 
for $p_{4}$ (Fig. 5b). A good agreement between the two is observed for all pressure points in all runs.
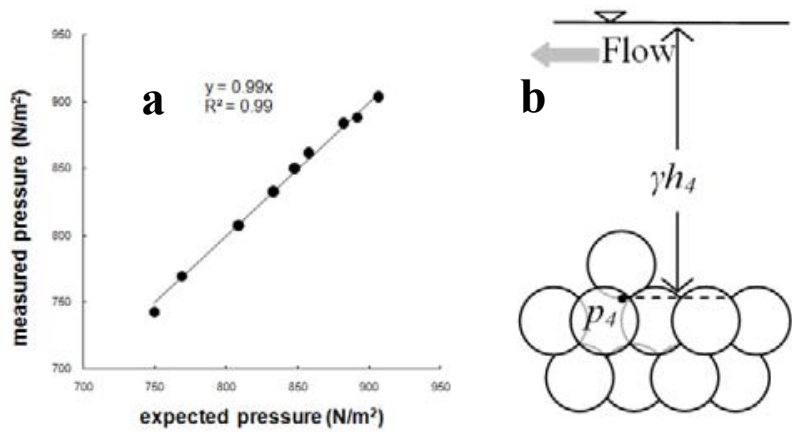

Figure 5. Comparison of measured and expected average (static) pressures. a) Measured vs. expected static pressures. b) Definition sketch of the flow depth relative to $p_{4}$ and the hydrostatic pressure. $\gamma$ is the specific weight of water and $h$ is the flow depth.

\subsection{Correlation between Individual Pressures}

Cross correlation functions (CCF) are obtained to investigate the similarities between two pressure time series as well as to detect the time lag between them. Figures 6-11 give the CCF's between individual pressures as a function of time $\operatorname{lag}(t)$.

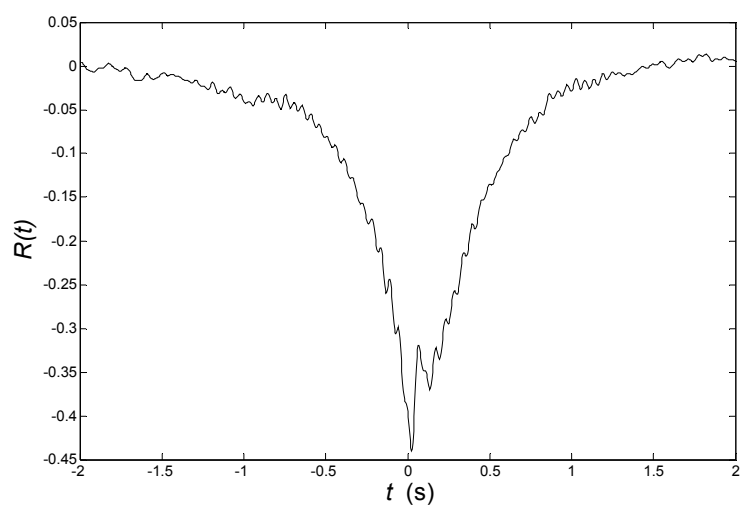

Figure 6. Cross correlation function between $p_{1}$ (front) and $p_{2}$ (back) from A2. Negative peak at time lag of $0.028 \mathrm{~s}$

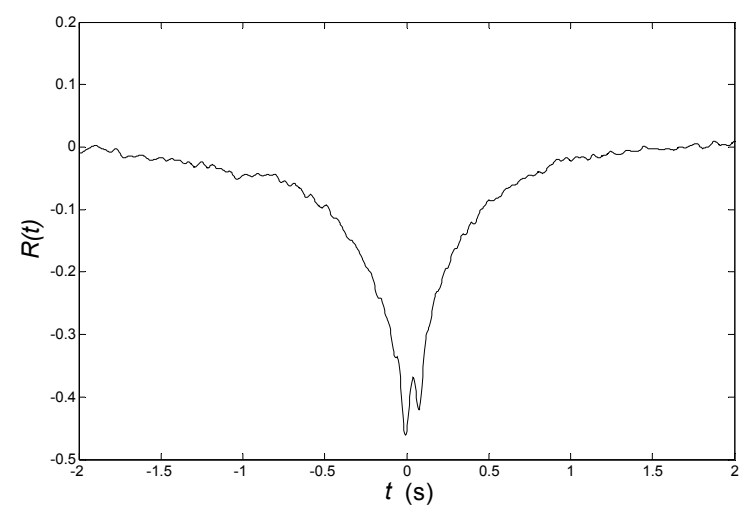

Figure 7. CCF between $p_{1}$ (front) and $p_{3}$ (top) from A2. Negative peak at lag of $-0.008 \mathrm{~s}$.

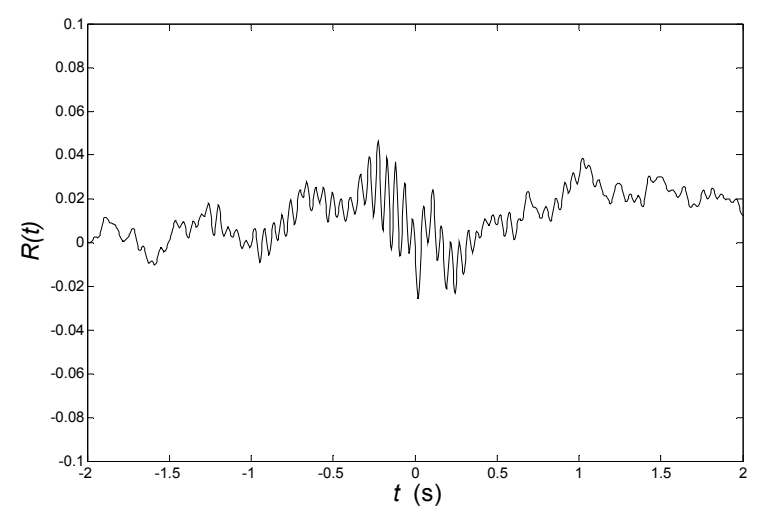

Figure 8. CCF betwen $p_{1}$ (front) and $p_{4}$ (bottom) from A2. Correlation is weak.

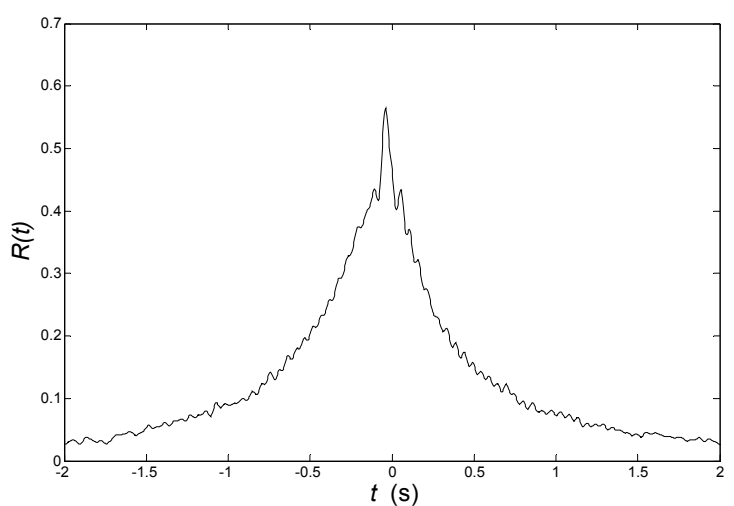

Figure 9. CCF between $p_{2}$ (back) and $p_{3}$ (top) from A2. Significant positive correlation is observed at the lag of -0.044 s.

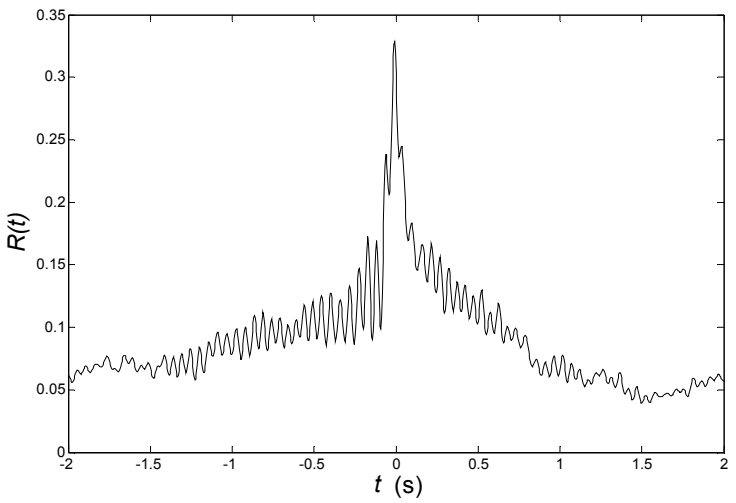

Figure 10. CCF between $p_{2}$ (back) and $p_{4}$ (bottom) from A2. A sensible peak is observed at the lag of $-0.012 \mathrm{~s}$.

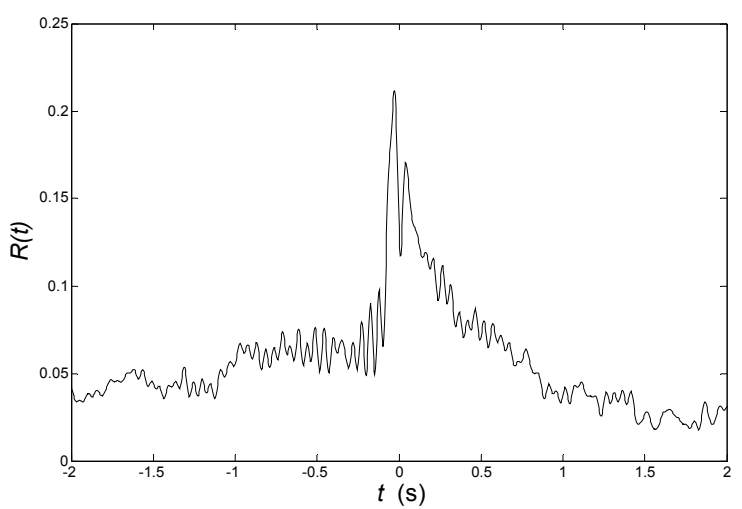

Figure 11. CCF between $p_{3}$ (top) and $p_{4}$ (bottom) from A2. Weak positive correlation is observed highest at the lag of $0.024 \mathrm{~s}$. 
Although they won't indicate the cause and effect relation between the events in the signals, some possible interpretations of the CCF plots are given as follows.

(i) $p_{1}$ and $p_{2}$, Fig. 6: Relatively strong negative correlation indicates the inverse relationship between the pressures in the front and back of the grain, origin of which the pressure in the front $(\sim$ positive lag).

(ii) $p_{1}$ and $p_{3}$, Fig. 7: Relatively strong negative correlation indicates the inverse relationship between the pressures in the front and top, cause of which is unclear due to negative lag. Also a second strong peak at lag $+0.076 \mathrm{~s}$ is apparent.

(iii) $p_{1}$ and $p_{4}$, Fig. 8: Very low $R(t)$ values indicate that there is very weak or no correlation between these two pressures.

(iv) $p_{2}$ and $p_{3}$, Fig. 9: Significant positive correlation is observed at the lag $-0.044 \mathrm{~s}$. This observation indicates that the flow processes that are causing high pressure on top of the particle (negative lag) are also very likely to be responsible for pressure peaks at the back. The same is also true for low pressures.

(v) $p_{2}$ and $p_{4}$, Fig. 10: A sensible peak is observed at the lag $-0.012 \mathrm{~s}$. Flow events that are moving towards the bed fast, are possibly causing what is observed here. A strong sweep (Willmarth, 1973) will increase the flow velocities around the ball decreasing the pressure in the bottom and back (see also Fig.6)

(vi) $p_{3}$ and $p_{4}$, Fig. 11: Weak positive correlation is observed highest at the lag $-0.024 \mathrm{~s}$.

It is observed that the pressures $p_{1}, p_{2}$ and $p_{3}$ are highly correlated with each other. In Figs. 8, 10 and 11 , the cross correlation functions fluctuate rhythmically. This is a sign of an oscillation in one or both signals. Possible cause of this is the vortex shedding around the test grain (i.e. oscillations in pressure signals $p_{2}$ and $p_{4}$.) as also apparent in the auto-correlation function plots of pressure signals shown in Fig. 11. Weak oscillations are observed in $p_{3}$ as well.

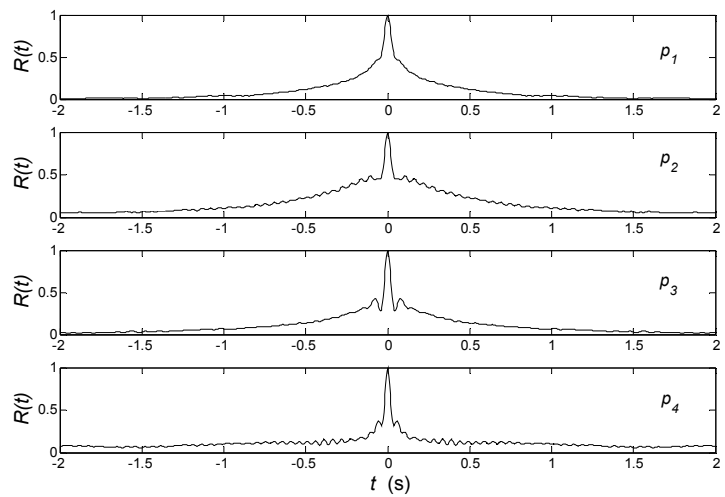

Figure 12. Auto correlation functions of pressures from run A2.

\subsection{Correlation between Pressure and Flow Velocity}

Synchronously measured local flow velocity components ( $u$ and $w$ respectively) and pressures acting on the test grain are analyzed. Fig. 13 presents the CCFs between the pressure signals and $u$. Fig. 14 shows CCFs between the pressures and $w$.

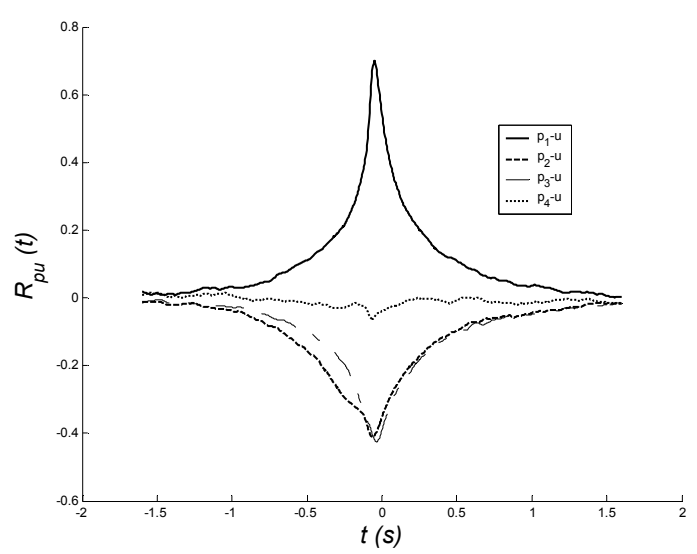

Figure 13. CCFs between the pressures and $u$ from run A1.

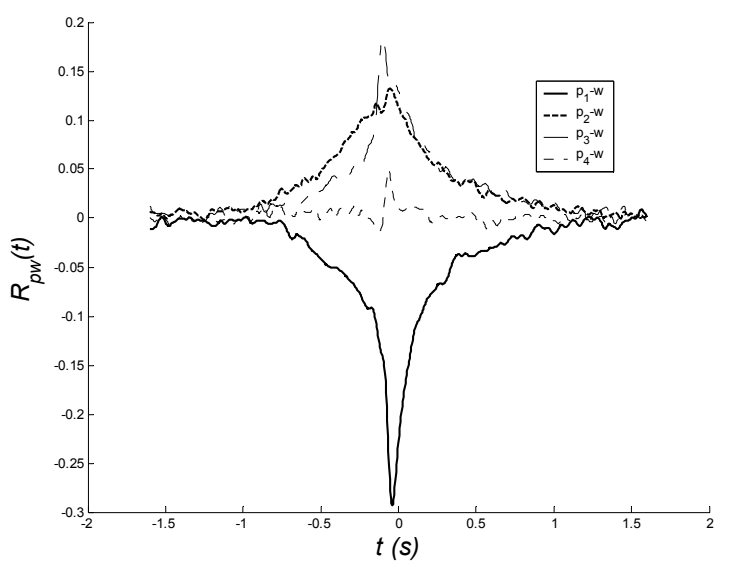

Figure 14. CCFs between pressures and $w$ from run A1.

According to the CCFs with respect to time lags shown in Fig. 13, $p_{2}$ and $p_{3}$ are inversely correlated with $u$ while as expected; $p_{1}$ has a strong positive correlation with $u$. This finding is consistent with the negative correlation between $p_{1}$ and $p_{2}$ as well as $p_{1}$ and $p_{3}$ we presented earlier (Figs. 6 and 7). The indication here is that the fast flow events (sweeps and outward interactions for example) that are causing an increase in $p_{1}$ have the opposite effect in $p_{2}$ and $p_{3}$. In another possible flow event, where a slow fluid parcel is ejected from the bed, $p_{1}$ will be low and $p_{2}$ and $p_{3}$ will increase due to same principles discussed above. On the other hand, $p_{4}$ was found to be not connected to $u$.

CCFs between pressures and $w$ (Fig. 14) suggest that there is noticeable negative correlation between $p_{1}$ and $w$. This assertion agrees with the interpretations we gave above that during a sweep like event when $w$ is towards the bed, $u$ and $p_{l}$ have high values. When $u$ is low and $w$ is positive (ejection) there is a decrease in $p_{1}$. 


\subsection{Pressures and Shear Stress}

The ratio of root-mean-square (rms) of pressure fluctuations, $p$ ', over the bed shear stress, $\tau_{b}$, with respect to particle Reynolds number, $R e^{*}$ is given in Fig. 15 for 9 flow conditions (see Table 1).

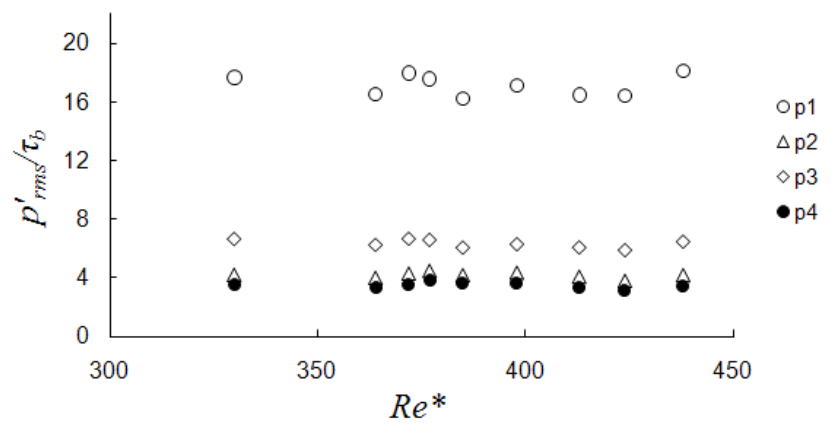

Figure 15. $p^{\prime}{ }_{r m s} / \tau_{b}$ vs. $R e^{*}$ plots from all runs.

An average value of 3 for this ratio is reported in the literature (Smart and Habersack, 2007). In our experiments, we obtained values close to 3 only for $p_{2}$ and $p_{4}$ (back and bottom pressures respectively). For $p_{1}$ and $p_{3}$ these ratios were near 18 and 7 respectively. These very high values indicate the inadequacy of the shear stress approaches in describing the flow induced forces.

In these experiments, there is a time delay between the flow velocities measured one diameter upstream of the instrumented grain and the pressures acting on it due to the distance between the LDV measurement volume and the grain location. The strong correlation we observed between $p_{1}$ and $u$ which is shown in Fig. 13 was utilized to estimate the time delay between the two signals (i.e. maximum of their CCF). Then the calculated time delays were taken into account in order to synchronize the local flow velocity and pressure time series $\left(p_{1}-p_{4}\right)$ for each run. Representative time series of synchronized $p_{1}$ and $u$ in this fashion from run $\mathrm{A} 2$ are shown in Fig 16.
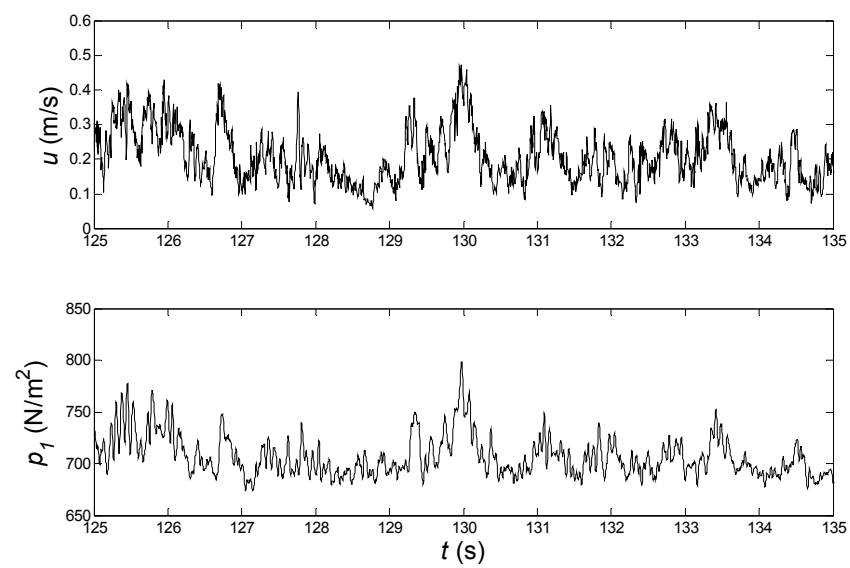

Figure 16. Time traces of the synchronized streamwise flow velocity, $u$ (top plot ) and the pressures acting on the front face of the particle, $p_{l}$ (bottom plot).

\subsection{Drag and Lift Forces}

Instantaneous forces were estimated from the measured instantaneous pressures by $D=$ $A\left(p_{1}-p_{2}\right)$ and $L=A\left(p_{4}-p_{3}\right)$, where $D$ is the drag force, $L$ is the lift force and $A$ is the projected are of the spherical test grain (Einstein and El Samni, 1949; Hofland et al., 2005; see the definition sketch and the insets in Fig. 1d). Note that positive $L$ is acting upwards, perpendicular to the flow direction.

CCFs between the local flow velocity components and the force components are investigated next. Figure 17 shows the plots of CFFs between " $u$ and $D$ " and " $w$ and $D$ ". The plots of CFFs between " $u$ and $L$ " and " $w$ and $L$ " are given in Fig. 18.

Results indicate a dependency of $D$ on $u$ and also on $w$ the former being stronger. This is consistent with findings of Hofland et al. (2005). Also we note that the lift force is correlated with $u$ to some degree (Fig. 18) contradicting the findings of Schmeeckle et al. (2007). One interesting observation is that the lift force shows a weak correlation with $w$ although the lag time corresponding to the peak in the CCF is about $0.1 \mathrm{~s}$ which is relatively high. This finding cannot be explained.

Average drag coefficient, $C_{D}$, varied between 0.8 and 1.3 for the flow conditions tested. Figure 19 below shows the estimated average $C_{D}$ values vs. $R e^{*}$. These $C_{D}$ values disagree with commonly assumed lower $C_{D}$ values for spheres in sediment research. However, $C_{D}$ values reported here agree with the recent findings of Schmeeckle et al. (2007).

Drag force momentarily reached up to 6 times the mean value. Lift force magnitude was observed to be momentarily having values up to nearly $50 \%$ of the submerged weight of the Teflon particle in both upward and downward directions. This indicates that the lift force fluctuations, despite the negligible mean value for the fully exposed grain configuration, can play a role in particle dislodgement as well as stabilization.

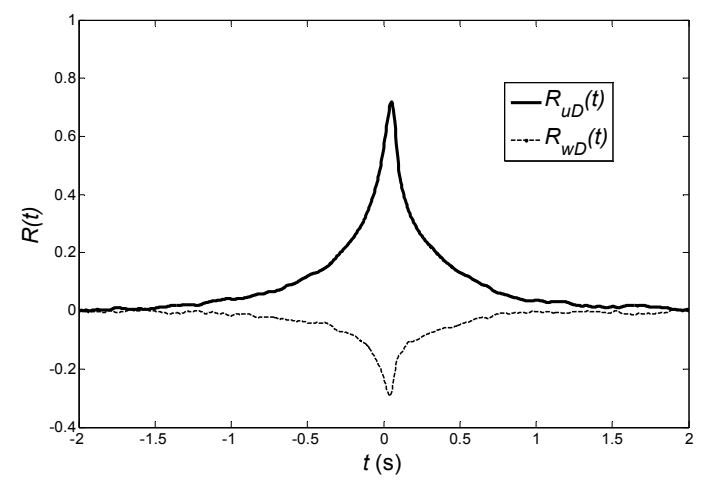

Figure 17. CCFs between $u$ and $D$ (bold line and) $w$ and $D$ (dashed line) from run A1 (peaks around $+0.052 \mathrm{~s}$ and $+0.044 \mathrm{~s}$ respectively) 


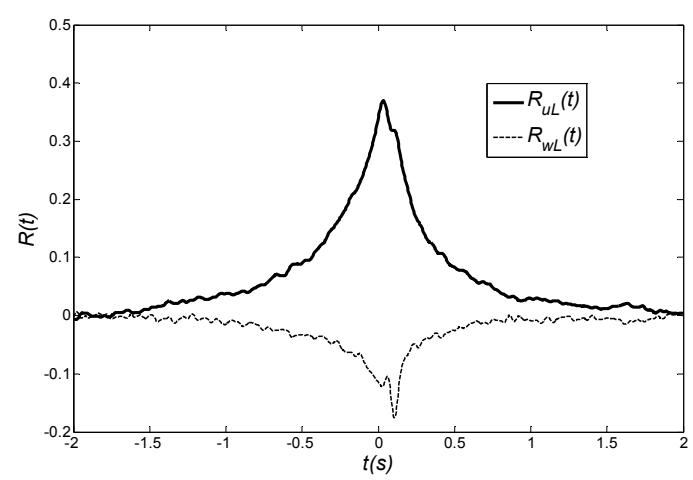

Figure 18. CCFs between $u$ and $L$ (bold line and) $w$ and $L$ (dashed line) from run A1 (peaks around $+0.032 \mathrm{~s}$ and +0.1 $\mathrm{s}$ respectively)

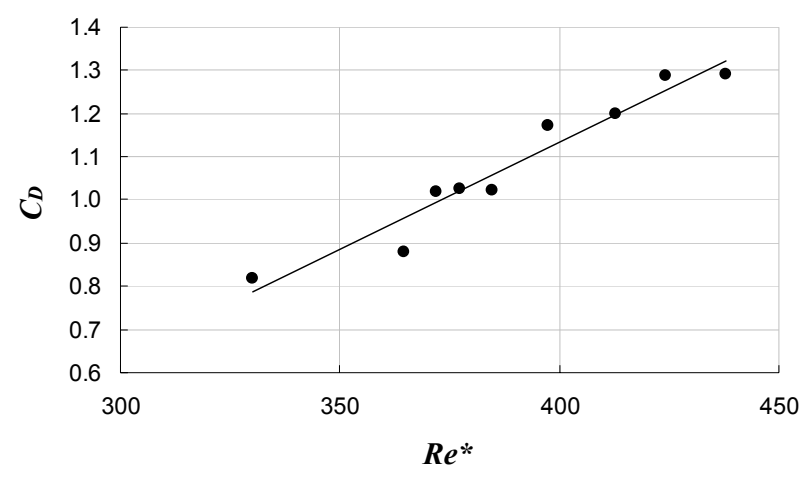

Figure 19. Average $C_{D}$ vs. $R e^{*}$ from all runs

\section{SUMMARY AND CONCLUSIONS}

In this work, an experimental method was described which was used to measure the near bed pressures together with the local flow velocity. Measurement uncertainties were explored in detail. The experimental data were analyzed to describe the features of the turbulent pressures and their relations to near bed velocity. Experimental results show that the instantaneous pressures acting in the front, back and the top of the grain are correlated to each other. These pressures are also strongly influenced by the local flow velocities.

Drag and lift forces were estimated from the measured pressure differences. Analysis of the force and velocity components suggests that the instantaneous drag and lift forces are both correlated to the near bed streamwise velocity, drag being most pronounced. Weak negative correlation was also observed between the vertical flow velocity component and the drag and lift forces. Results presented here are very useful for more realistic incipient motion models although instantaneous coupling of drag and lift forces as well as their temporal variations and durations need to be further investigated.

\section{ACKNOWLEDGEMENTS}

The support of the National Science Foundation (EAR-0439663 and EAR-0738759) for this study is gratefully acknowledged. Also the writers thank Mr. Colin Steward for performing flume vibration tests.

\section{REFERENCES}

Dancey, C. L., Diplas, P., Papanicolaou, A., and Bala, M. (2002). "Probability of Individual Grain Movement and Threshold Condition" J. Hydraul. Eng., ASCE, 128(12), 1069-1075.

Diplas P., Dancey C. L., Celik A. O., Valyrakis M., Greer K., and Akar T. (2008). "The role of impulse on the initiation of particle movement under turbulent flow conditions" Science, 322, 717-720

Einstein, H. A., and El-Samni, E. A. (1949). "Hydrodynamic forces on a rough wall” Rev. Mod. Phys., 21(3), 520524.

Hofland, B., Booij, R., and Battjes, J. 2005. Measurement of Fluctuating Pressures on Coarse Bed Material, Journal of Hydraulic Engineering, ASCE, Vol.131, No.9, 770-781

Lu, S. S., and Willmarth, W. W. 1973. Measurements of the Structure of the Reynolds Stress in a Turbulent Boundary Layer, Journal of Fluid Mechanics, Vol. 60, 481511

Nelson, J., Shreve, R. L., McLean, S. R., and Drake, T. G. 1995. Role of Near-bed Turbulence Structure in Bedload Transport and Bed-form Mechanics, Water Resources Research, AGU, Vol.31, No.8, 2071-2086

Papanicolaou, A. N., Diplas, P., Evaggelopoulos, N., and Fotopoulos, S. 2002. Stochastic Incipient Motion Criterion for Spheres under Various Bed Packing Conditions, Journal of Hydraulic Engineering, ASCE, Vol.128, No.4, 369-380

Schmeeckle, M. W., Nelson J. M., and Shreve R. L. 2007. Forces on Stationary Particles in Near-bed Turbulent Flows, Journal of Geophysical Research, AGU, Vol.112, F02003

Smart G. M., and Habersack H. M. 2007. Pressure Fluctuations and Gravel Entrainment in Rivers, Journal of Hydraulic Research, IAHR, Vol.45, No.5, 661-673

Stewart, C. 2009. Environmental and Pump Induced Vibrations in Test Flumes. Technical Report, BEHL at Virginia Tech

Stoesser, T., Fröhlich, J., Rodi W. (2007). Turbulent OpenChannel Flow over a Permeable Bed. Proc. 32nd IAHR Congress, Venice, 2007.

Yoshida, A., Tamura Y. and Kurita, T. 2001. Effects of bends in a tubing system for pressure measurement, J. Wind Eng. Ind. Aerodyn. 89 (20) 1701-1716. 